\title{
FATORES CONTROLADORES DA COMPRESSIBILIDADE DE UM ARGISSOLO VERMELHO-AMARELO DISTRÓFICO ARÊ NICO E DE UM LATOSSOLO VERMELHO DISTRÓFICO TÍPICO. II - GRAU DE SATURAÇÃO EM ÁGUA ${ }^{(\mathbf{1})}$
}

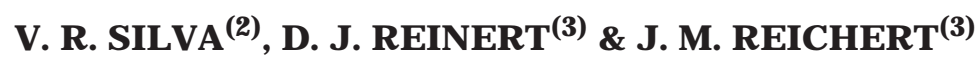

\begin{abstract}
RESUMO
O teor de água no solo constitui fator determinante na capacidade de um solo em resistir à compactação por pisoteio animal ou tráfego de máquinas. 0 objetivo deste estudo foi avaliar o efeito do teor de água (saturação dos poros em água) nos valores de pressão de preconsolidação $\left(\sigma_{p}^{\prime}\right)$ e no índice de compressão $\left(C_{c}\right)$, para dois solos com texturas contrastantes, em duas profundidades, cultivados sob sistema plantio direto e convencional. Amostras indeformadas $(5,35 \mathrm{~cm}$ de diâmetro por $2 \mathrm{~cm}$ de altura) foram coletadas na camada superficial $(0-2 \mathrm{~cm})$ e na camada de $10-12 \mathrm{~cm}$, durante 0 ano agrícola de 1997/1998, num Argissolo Vermelho-Amarelo distrófico arênico $\left(89 \mathrm{~g} \mathrm{~kg}^{-1} \mathrm{de}\right.$ argila), localizado no município de Santa Maria (RS) $\left(29^{\circ} 45^{\prime}\right.$ latitude sul e $53^{\circ} 42^{\prime}$ longitude oeste) e de um Latossolo Vermel ho distrófico típico (467 g kg-1 de argila), localizado no município de I birubá (RS) $\left(28^{\circ} 30^{\prime}\right.$ latitude sul e $53^{\circ} 30^{\prime}$ longitude oeste). Para cada tipo de solo e condição de manejo, amostras indeformadas foram coletadas em diferentes épocas para obter variação natural de grau de saturação e, ainda, algumas amostras foram saturadas e equilibradas em laboratório para obter uma ampla variação de grau de saturação. Para cada solo, sete classes de grau de saturação foram estabelecidas (<15; 16 a 30; 31 a 45; 46 a 60; 61 a 75; 76 a 90 e 91 a 100\%). O ensaio de compressão uniaxial foi realizado com aplicação sucessi va de cargas estáticas de 12,5; 25; 50; 100; 200; 400 e 800 kPa, durante cinco minutos cada. Os valores de densidade do solo também foram distribuídos em classes, de acordo com a disponi bilidade de amostras. Os valores da $\sigma_{p}^{\prime}$ na camada superficial do Argissolo sob plantio direto foram superiores nos graus de saturação até $30 \%$ em relação aos demais graus de saturação. Para o Latossolo, na faixa de densidade do solo de 1,30 a 1,45 $\mathrm{Mg} \mathrm{m}^{-3}$, o aumento do
\end{abstract}

\footnotetext{
(1) Parte da Tese de Mestrado do primeiro autor, apresentada ao Programa de Pós-Graduação em Agronomia, Universidade Federal de Santa Maria - UFSM. Pesquisa executada com recursos do CNPq/FINEP (Projeto PRONEX 60/97). Recebido para publicação em novembro de 2000 e aprovado em julho de 2001.

(2) Doutorando do Programa de Pós-Graduação em Agronomia, UniversidadeFederal deSanta Maria - UFSM. CCR-SOLOS, Campus, CEP 97105-900 Santa Maria (RS). E-mail: silvavr@bol.com.br

(3) Professor Titular do Departamento de Solos, UFSM. Bolsista do CNPq. E-mails: dalvan@ccr.ufsm.br; reichert@ccr.ufsm.br
} 


\begin{abstract}
grau de saturação de 46-60\% para 61-75\% representou dimi nuição nos valores da $\sigma_{p}^{\prime}$ de 1,6 vez na condição de plantio direto, nas duas profundidades, e de 2,4 vezes na camada superficial da área com manejo convencional. Os valores de $\sigma_{p}^{\prime}$ diminuíram de forma logarítmica com o aumento do grau de saturação.
\end{abstract}

\author{
Termos de indexação: compactação do solo, plantio direto, índice de compressão, \\ pressão de preconsolidação.
}

\author{
SUMMARY: FACTORS CONTROLLING COMPRESSIBILITY OF A \\ PALEUDALF AND A HAPLORTOX SOIL. II - DEGREE OF \\ WATER SATURATION
}

\begin{abstract}
Soil water content is one of the determining factors in control ling compaction caused by animal trampling or machinery traffic. The objective of this study was to evaluate the effect of water content (pore water saturation) on preconsolidation pressure $\left(\sigma_{p}^{\prime}\right)$ and compression index $\left(C_{c}\right)$ values, for two soils with contrasting textures, at two depths, cultivated under no tillage and conventional tillage. Undisturbed soil samples $(5.35 \mathrm{~cm}$ of diameter for $2 \mathrm{~cm}$ of height) werecollected in thesurfacelayer $(0$ to $2 \mathrm{~cm}$ ) and in the 10 to $12 \mathrm{~cm}$ layer from, during theagricultural year of 1997/ 1998, for a Paleudalf ( $89 \mathrm{~g} \mathrm{~kg}^{-1}$ of clay), located in Santa Maria (RS), Brazil (29 $45^{\prime}$ latitude South and $53^{\circ} 42^{\prime}$ longitude West), and a Haplortox (467 g kg-1 of clay), located in I bi rubá, RS, Brazil ( $28^{\circ} 30^{\prime}$ latitudeSouth and $53^{\circ}$ 30 ' longitude West). To obtain a wide range of saturation degree for each soil type and management system, undisturbed soil samples were collected at different times to obtain natural variation of saturation degre. Some samples were saturated and equilibrated in the laboratory. For each soil, seven classes of saturation degree were established: <15; 16 to $30 ; 31$ to $45 ; 46$ to $60 ; 61$ to $75 ; 76$ to 90 and 91 to $100 \%$. The uniaxial compression test was done using successi ve static loads of $12.5,25,50,100,200,400$ and $800 \mathrm{kPa}$, during five minutes each load. The values of bulk density werealso classified in classes, based on sample availability. The values of the $\sigma_{p}^{\prime}$, for the surface layer of the Paleudalf under no tillage, were greater for saturation degrees up to $30 \%$, compared to the other saturation degrees. F or the Haplortox, for thebulk density rangefrom 1.30 to $1.45 \mathrm{Mg} \mathrm{m}^{-3}$, theincrease in the degree of saturation from $46-60 \%$ to $61-75 \%$ represented a decreasein $\sigma_{p}^{\prime}$ values of 1.6 times for the no tillage, at the two depths, and of 2.4 times for the surface layer under conventional tillage. The values of $\sigma_{p}^{\prime}$ decreased in a logarithmic way, with the increase of the saturation degree
\end{abstract}

Index terms: soil compaction, no tillage, compression index, preconsolidation pressure

\section{INTRODUÇÃO}

A compactação de solos agrícolas tem sido estudada correlacionando sistemas de cultivo que provocam maiores ou menores impactos sobre o solo, porém poucos estudos abordam o processo de compactação de sol os. O solo compacta-se pela ação de forças externas que tendem a deformá-lo, com redução do espaço poroso. Tais forças podem ser oriundas do pisoteio animal ou do tráfego de máquinas sobre o solo (Soane \& van Ouwerkerk, 1994). O efeito da compactação no solo, na maioria das vezes, é negativo para a produção de plantas, interferindo na disponibilidade de água e oxigênio, no aumento da densidade do solo e da resistência que o solo oferece à penetração de raízes, levando à redução do crescimento das plantas e à degradação física do solo (Håkansson \& Woorhees, 1997; Camargo \& Alleoni, 1997; Pabin et al., 1998).

A curva de compressão do solo representa a redução do índice de vazios de um solo, conforme o aumento da pressão aplicada sobre ele (Holtz \& Kovacs, 1981). Essa curva divide-se em duas partes (Holtz \& K ovacs, 1981; Kondo \& Dias únior, 1999b): (a) a curva de recompressão ou compressão secundária, que representa pressões aplicadas ao sol o que não provocam compactação adicional, e (b) a reta virgem de compressão, onde a capacidade de suporte de carga do solo é vencida e ocorrem deformações elásticas, não-recuperáveis. O ponto que limita essas duas porções da curva de compressão échamado de pressão de preconsoli dação $\left(\sigma_{p}^{\prime}\right)$, sendo um indicativo da capacidade de suporte do solo e pode ser considerado como um indicador 
quantitativo da sustentabilidade estrutural dos sol os (Kondo \& Dias J unior, 1999a). A inclinação da reta virgem de compressão é chamada de índice de compressão $\left(C_{c}\right)$ e demonstra a susceptibilidade do solo à compactação (Larson et al., 1980; Holtz \& Kovacs, 1981).

No processo de compactação do solo, a determinação da $\sigma_{p}^{\prime}$ e do $C_{c}$ tem grande importância na adequação de máquinas que exerçam menores pressões sobre o solo e, se o valor da $\sigma_{p}^{\prime}$ for vencida, pode-se estimar a compactação adicional (K ondo \& Dias J unior, 1999b). Por outro lado, para o sistema radicular das plantas desenvolver-se, é necessário que as raízes vençam a $\sigma_{p}^{\prime}$ do solo (Kondo \& Dias J unior, 1999c).

Para três Latossolos, Kondo \& Dias J unior (1999a) verificaram que o aumento da umidade do solo provocou o deslocamento da reta virgem de compressão para uma região de menor pressão, indicando um aumento da susceptibilidade do solo à compactação (maior $\mathrm{C}_{\mathrm{c}}$ ), corcordando com resultados de Larson et al. (1980).

Reinert (1990), trabalhando em solo siltoso, verificou maior $\sigma_{p}^{\prime}$ no sistema decultivo plantio direto comparado com o sistema convencional de manejo. Esse efeito pode estar relacionado com os maiores val ores de densidade do sol o encontrados na condição de plantio direto. Para Kondo \& DiasJ unior (1999a), o solo cultivado anualmente com culturas anuais apresentou maior $\sigma_{p}^{\prime}$ do que os de pastagem e mata natural, fato que pode ser atribuído à degradação da estrutura do solo pelo cultivo intensivo.

A condição de umidade do solo é fator determinante da capacidade de suporte de carga do solo. Quando seco, o sol o ébastante coeso eapresenta grande resistência à compactação, mas, à medi da que ele vai umedecendo, ocorre a formação de um pequeno filme de água entre os agregados do solo, facilitando o deslocamento e o rearranjamento das partículas sólidas da matriz do solo, diminuindo a resistência do solo à compactação (Hillel, 1980).

Estudos encontrados na literatura mostram que a $\sigma_{p}^{\prime}$ decresce exponencial mente com o aumento do teor de água no solo (Dias J unior, 1994; Kondo \& Dias J unior, 1999a; Silva et al., 1999). Todavia, são necessárias mai ores investigações sobrea influência do teor de água em outros tipos de sol os e sistemas demanejo, principalmenteno sistema plantio direto.

Neste estudo, testou-se a hipótese de que a umidade do sol o e o tipo de manejo do sol o afetam os valores de resistência às deformações plásticas, ou seja, a pressão de preconsolidação e o índice de compressão do solo. Desse modo, o objetivo deste trabal ho foi determinar a pressão de preconsol idação e o índice de compressão de dois sol os com texturas e manejos contrastantes, em duas profundidades, e avaliar suas relações com o grau de saturação com água para cada solo.

\section{MATERIAL E MÉTODOS}

Utilizaram-se dois tipos de solos: um Argissolo Vermelho-Amarelo distrófico arênico (Paleudalf) e um L atossol oVermel ho distrófico típico (Haplortox), cuja local ização, histórico de manejo eresultados de al gumas propriedades físicas e carbono orgânico do solo estão descritos em Silva et al. (2002).

As amostras de solo foram coletadas no campo ao acaso, buscando-se variação natural no grau de saturação do solo. Utilizaram-se 260 amostras, coletadas em anéis de $5,36 \mathrm{~cm}$ de diâmetro por $2,0 \mathrm{~cm}$ de altura, em duas profundidades (0-2 e 10$12 \mathrm{~cm}$ ), durante os meses de novembro de $1997 \mathrm{e}$ junho de 1998.

Para obter a variação no grau de saturação, foram utilizadas diferentes estratégias: (a) amostragem em diferentes épocas; (b) saturação de al gumas amostras em água durante 24 horas e submetidas à sucção em mesa de tensão nos potenciais de -1 e $-6 \mathrm{kPa}$ e em câmaras de Richards nos potenciais de -33, -100 e -500 kPa, e (c) col ocação de amostras em caixas de papelão com pequenos orifícios para que a redução de umi dade ocorresse de maneira lenta e de forma homogênea, obtendo amostras com baixo grau de saturação $\left(S_{r}\right)$. Os val ores de $S_{r}$ dos dois sol os foram distribuídos em sete classes (<15; 16a 30; 31 a 45; 46 a 60; 61 a 75; 76 a 90 e91 a 100\%). As distintas dasses foram definidas "a posteriori", conforme a variação nos val ores de $D_{s}$ e $S_{r}$ e a existência de um número razoável de amostras para cada classe estabel ecida.

O ensaio de compressão uniaxial (NBR-12007/90, Associação Brasileira de Normas Técnicas, 1990), o cál culo do índice de compressão $\left(C_{c}\right)$ e da pressão de preconsolidação $\left(\sigma_{p}^{\prime}\right)$ foram determinados pelo método de Casagrande (Holtz \& Kovacs, 1981; NBR-12007/90), podendo maiores detalhes ser encontrados em Silva et al. (2002).

Considerou-se o model ofatorial com delineamento inteiramente casualizado para comparações de médias entre manejos, profundidades, classes de grau de saturação e tipo de solo, utilizando a Diferença Mínima Significativa (DMS) com $\mathrm{P} \leq 0,05 \%$ para identificar médias diferentes entre si, utilizando o pacote estatístico SAS (SAS, 1985).

\section{RESULTADOS E DISCUSSÃO}

Na superfície do Argissol o sob plantio direto, os valores da $\sigma_{p}^{\prime}$ foram superiores nos graus de saturação $\left(\mathrm{S}_{r}\right)$ até $30 \%$ em relação aos demais $\mathrm{S}_{r}$. As cargas já aplicadas ao sol o, quelevaram a uma faixa de densidade do solo $\left(D_{\mathrm{s}}\right)$ entre 1,46 e $1,60 \mathrm{Mg} \mathrm{m}^{-3}$ foram suficientemente grandes para manter uma resistência até o início da deformação plástica de $67,9 \mathrm{kPa}$, mesmo com S r próximo a 90\% (Quadro 1). 
Para o Latossolo, na faixa de $D_{s}$ de 1,30 a $1,45 \mathrm{Mg} \mathrm{m}^{-3}$, o aumento do $\mathrm{S}_{\mathrm{r}}$ de 46 a $60 \%$ para 61 a $75 \%$ representou diminuição nos valores da $\sigma_{p}^{\prime}$ de 1,6 vez na condição de plantio direto, nas duas profundidades, e de 2,4 vezes na camada superficial da área com manejo convencional (Quadro 1). Na camada de 10-12 cm, o Argissolo sob plantio direto apresentou um estado de compactação mais el evado $\left(D_{s}\right.$ de 1,61 a $1,75 \mathrm{Mg} \mathrm{m}^{-3}$ ), sendo necessário um $\mathrm{S}_{\mathrm{r}}$ mais el evado para diminuir a resistência do solo às deformações plásticas, o mesmo ocorrendo para o Latossolo.

No manejo convencional do Argissolo, a degradação da estrutura e a redução da matéria orgânica (Silva et al., 2002) refletiram-se, em geral, em baixos valores da $\sigma_{p}^{\prime}$ na camada superficial. Com $\mathrm{S}_{\mathrm{r}}$ menor que $15 \%$, o Argissolo apresentou o maior valor da $\sigma_{p}^{\prime}$, chegando a val ores em torno de $180 \mathrm{kPa}$. Contudo, nas demais classes de saturação, os val ores de $\sigma_{p}^{\prime}$ foram cerca de sete vezes mais baixos, ficando em torno de $25 \mathrm{kPa}$. Na figura la, para a faixa de maior $D_{s}\left(1,68\right.$ a $\left.1,72 \mathrm{Mg} \mathrm{m}^{-3}\right)$, a $\sigma_{p}^{\prime}$ decresceu rapidamente até à saturação de $30 \%$. Na menor faixa de $D_{s}\left(1,30\right.$ a 1,40 $\left.\mathrm{Mg} \mathrm{m}^{-3}\right)$, o decréscimo da $\sigma_{p}^{\prime}$ ocorreu de forma mais acentuada e estabilizou-se em torno de $60 \mathrm{kPa}$ a partir da saturação de $40 \%$. Da mesma maneira para o Latossolo, nas faixas de menores $D_{s}$, os pontos foram deslocados para baixo e para a esquerda, indicando menor resistência do que quando o estado de compactação era mais el evado (Figura 1b). De maneira geral, no sistema plantio direto, o Latossolo apresentou maior capacidade de suporte atéo início da deformação plástica nos graus de saturação menores que $60 \%$, nas duas profundidades, quando comparado ao Argissolo.
Para isolar o efeito do estado de compactação nas curvas de compressão, utilizou-se a estratégia de normalizar as curvas de compressão, ou seja, dividir os valores deíndice de vazios no final de cada estádio de pressão aplicada pelo índice de vazios inicial de cada amostra. Então, as curvas de compressão normalizadas (Figura 2) foram deslocadas para a esquerda e para baixo, conforme o aumento do grau de saturação, concordando com outros autores (Larson et al., 1980; Culley \& Larson, 1980; Dias) unior, 1994; Kondo \& Dias J unior, 1999a,b). Nas faixas de $\mathrm{S}_{\mathrm{r}}$ de 46 a $60 \%$ e de 61 a $75 \%$, esse deslocamento foi mais acentuado, indicando uma susceptibilidade a deformações plásticas nessas faixas de $\mathrm{S}_{\mathrm{r}}$.

Para um $\mathrm{S}_{\mathrm{r}}$ similar, o Latossol o apresentou maior capacidade de suportar cargas até o início da deformação plástica queoArgissolo. A partir do grau de saturação acima de $60 \%$, oArgissolo, com estado de compactação mais el evado, teve maiores valores de $\sigma_{\mathrm{p}}^{\prime}$. Essefato podeser atribuído à granulometria dos solos, pois, no solo argiloso, a resistência mecânica do solo às deformações em condições de menor umidade é conferida pela mai or coesão entre as partículas sólidas do que em solo arenoso, resultados que concordam com os de Kondo \& Dias J unior (1999a), que encontraram maior resistência a deformações plásticas (maior $\sigma_{p}^{\prime}$ ) quanto maior o teor de argila. No estado de compactação mais elevado, o efeito do $S_{r}$ é maior para a $\sigma_{p}^{\prime}$ e menor para o $C_{c}$ (Figura 3). Já no menor estado de compactação, o $S_{r}$ tem efeito na $\sigma_{p}^{\prime}$ e no $C_{c}$.

Os valores máximos de $\mathrm{C}_{\mathrm{c}}$ ocorreram nos graus de saturação menores que 31 \% (Quadro 2), na faixa

Quadro 1. Valores médios de pressão de preconsolidação $\left(\sigma_{p}^{\prime}\right)$ para os dois solos, manejos e profundidade estudados, divididos em faixas de densidade do solo, considerando o grau de saturação

\begin{tabular}{|c|c|c|c|c|c|c|c|c|c|}
\hline \multirow{2}{*}{ Solo } & \multirow{2}{*}{ Manejo } & \multirow{2}{*}{ Profundidade } & \multicolumn{7}{|c|}{ Grau de saturação (\%) } \\
\hline & & & $<15$ & $16-30$ & $31-45$ & $46-60$ & $61-75$ & $76-90$ & 91-100 \\
\hline & & $\mathrm{cm}$ & & & . & $\mathrm{kPa}$ & 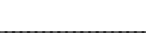 & 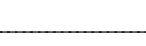 & 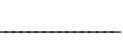 \\
\hline \multirow[t]{4}{*}{ Argissolo } & Direto & $0-2^{(2)}$ & 160,2 a & 136,4 a & $72,4 \mathrm{~b}$ & $69,6 \mathrm{~b}$ & $70,4 \mathrm{~b}$ & $67,9 \mathrm{~b}$ & $-(4)$ \\
\hline & & $10-12^{(3)}$ & $139,0 \mathrm{a}$ & $156,0 \mathrm{a}$ & $156,2 \mathrm{a}$ & $141,2 \mathrm{a}$ & 119,3 a & $112,1 a$ & $87,0 \mathrm{a}$ \\
\hline & Convencional & $0-2^{(1)}$ & 179,9 a & $26,2 \mathrm{~b}$ & $21,6 \mathrm{~b}$ & $27,6 \mathrm{~b}$ & - & $21,7 \mathrm{~b}$ & $-(4)$ \\
\hline & & $10-12^{(3)}$ & 132,2 a & - & 155,7 a & 159,9 a & $75,3 \mathrm{~b}$ & $103,5 a b$ & 155,2 a \\
\hline \multirow[t]{4}{*}{ Latossolo } & Direto & $0-2^{(1)}$ & 176,8 a & - & $159,4 a b$ & 128,7 b & $78,2 \mathrm{c}$ & $88,6 \mathrm{c}$ & $76,6 \mathrm{c}$ \\
\hline & & $10-12^{(1)}$ & $86,9 a b$ & $140,4 a b$ & 152,8 a & 160,7 a & $98,7 a b$ & $76,4 a b$ & $53,9 b$ \\
\hline & Convencional & $0-2^{(1)}$ & - & 174,3 a & $151,8 \mathrm{a}$ & 153,4 a & $63,5 b$ & $84,9 b$ & $73,6 \mathrm{~b}$ \\
\hline & & $10-12^{(2)}$ & - & $176,1 \mathrm{a}$ & - & 165,6 a & 149,3 a & $108,2 \mathrm{a}$ & 100,8 a \\
\hline
\end{tabular}



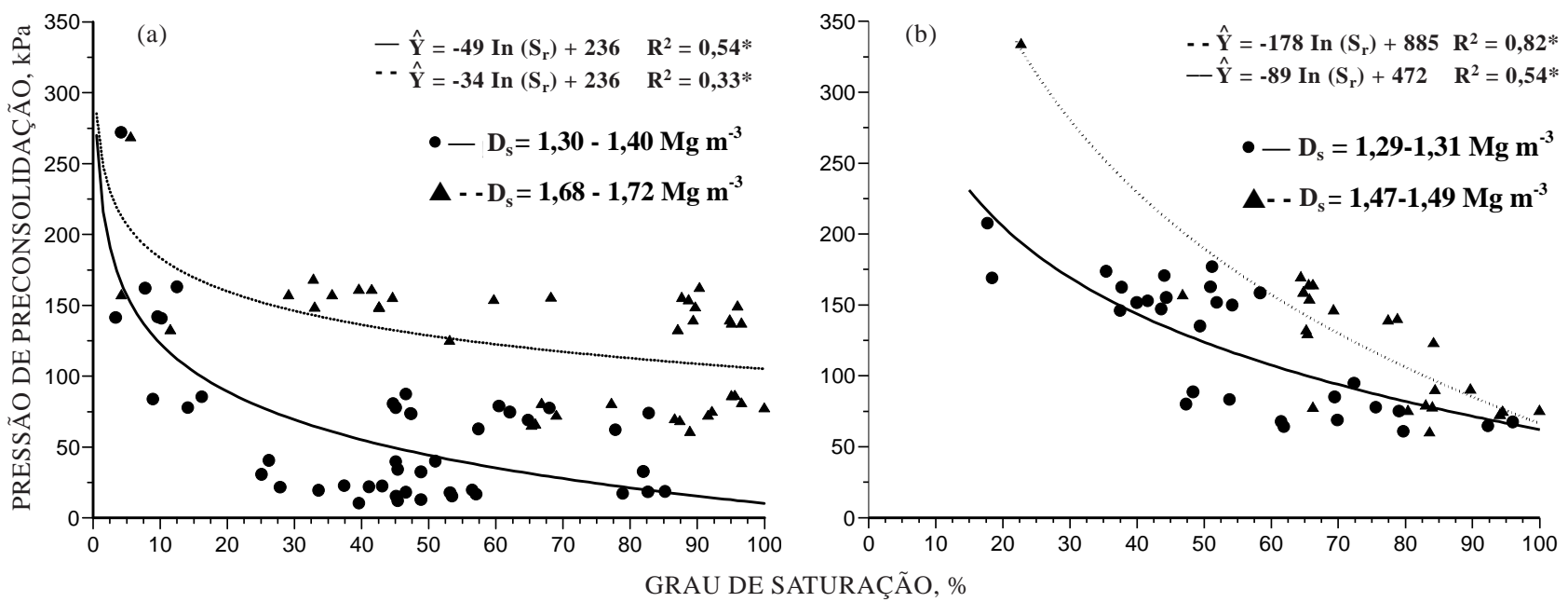

Figura 1. Valores de pressão de preconsolidação $(\mathrm{kPa})$, considerando o grau de saturação (\%) para duas faixas de $\rho_{s^{\prime}}$ (a) Argissolo e (b) Latossolo.

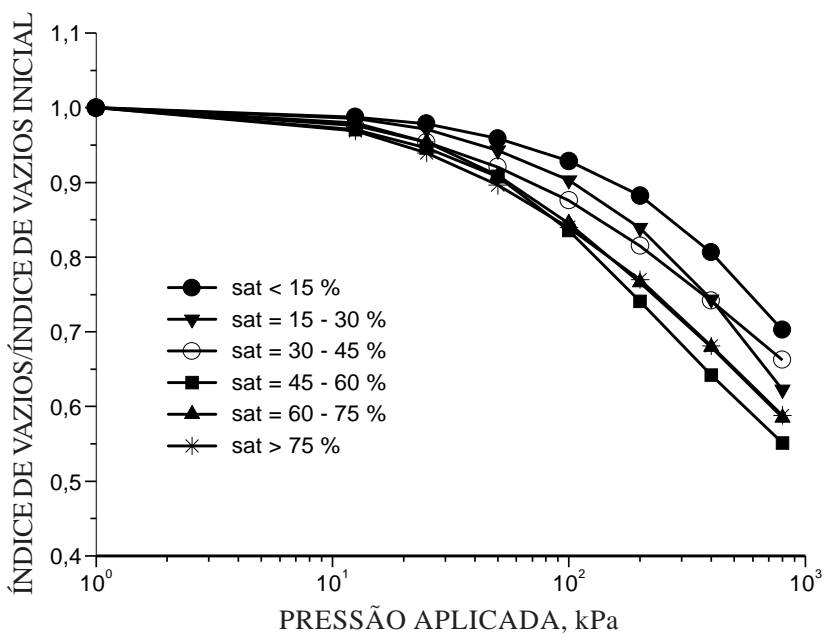

Figura 2. Curva de compressão normalizada do Argissolo, considerando diferentes graus de saturação.

de $\mathrm{D}_{\mathrm{s}}$ variando de 1,31 a $1,45 \mathrm{Mg} \mathrm{m}^{-3}$, para o plantio convencional, ede 1,46 a 1,60 $\mathrm{Mg} \mathrm{m}^{-3}$, para o plantio direto, no Argissolo. Com o estado de compactação mais elevado, $D_{s}$ de 1,61 a $1,75 \mathrm{Mg} \mathrm{m}^{-3}$, os maiores valores de $\mathrm{C}_{c}$ ocorreram em saturações mais elevadas, 46 a $60 \%$, no plantio direto, e 61 a $75 \%$, no plantio convencional. Esse fato deve estar associado ao atrito entre as partículas sól idas do sol o que, em estado de compactação mais el evado, é suficientemente grande para limitar o deslocamento e rearranjamento dessas partículas, sendo necessárias maiores quantidades de água para facilitar o deslocamento das partículas de solo.

A redução do $C_{c}$ máximo $(0,37)$ da camada superficial no Latossolo para o $\mathrm{C}_{\mathrm{c}}$ mínimo $(0,17)$ dessa camada foi de mais de 2 vezes e, para a

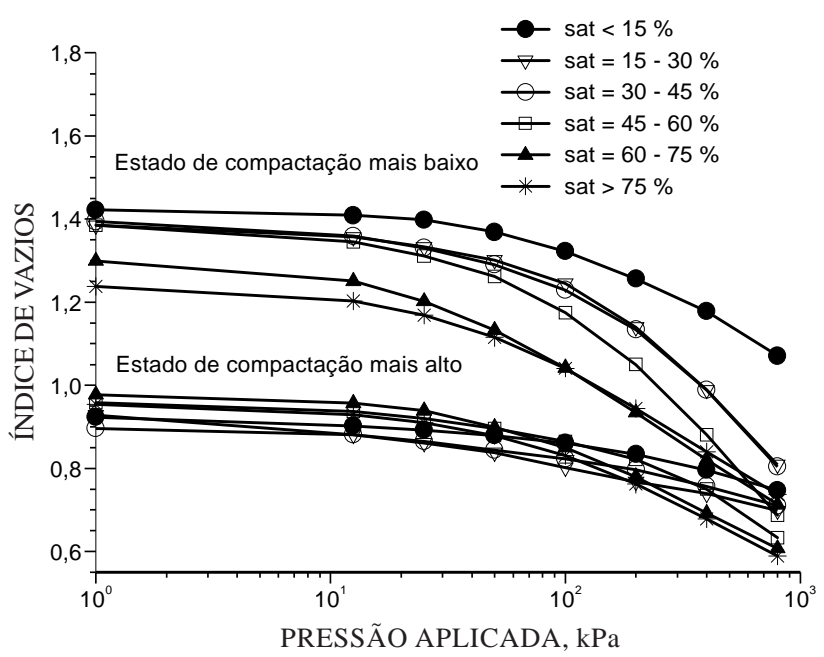

Figura 3. Curvas de compressão do Latossolo para dois estados iniciais de compactação, considerando diferentes graus de saturação.

profundidade de $10-12 \mathrm{~cm}$, a redução foi cerca de 4,4 vezes menos $(0,44$ para 0,10$)$, ambas no sistema plantio direto (Quadro 2). Nesse tipo de solo, quando seco, a resistência dada pel os pontos de contato entre os agregados é bastante grande para resistir às deformações. Na medida em queocorrea formação de uma lâmina de água envolvendo os agregados, esses tornam-sebastantesusceptíveis a grandes deformações.

Os valores máximos de $C_{c}$ no $L$ atossol o ocorreram em saturações intermediárias, enquanto, no Argissolo, nas menores saturações. Essa diferenciação entre os dois tipos de solos, segundo Larson et al. (1980), pode ser atribuída à granulometria. Os autores encontraram aumento linear do $\mathrm{C}_{\mathrm{c}}$ com oteor de argila de até $330 \mathrm{~g} \mathrm{~kg}^{-1}$, estabilizando a partir desse teor. 
Quadro 2. Valores médios de índice de compressão $\left(\mathrm{C}_{\mathrm{c}}\right)$ para os dois solos, manejos e profundidades estudados, divididos em faixas de densidade do solo, considerando diferentes graus de saturação

\begin{tabular}{|c|c|c|c|c|c|c|c|c|c|}
\hline \multirow{2}{*}{ Solo } & \multirow{2}{*}{ Manejo } & \multirow{2}{*}{ Profundidade } & \multicolumn{7}{|c|}{ Grau de saturação (\%) } \\
\hline & & & $<15$ & $16-30$ & 31-45 & $46-60$ & $61-75$ & 76-90 & 91-100 \\
\hline \multirow{3}{*}{ Argissolo } & & $\mathrm{cm}$ & & & & & & & \\
\hline & Direto & $\begin{array}{c}0-2^{(2)} \\
10-12^{(3)}\end{array}$ & $\begin{array}{l}0,19 a \\
0,09 a b\end{array}$ & $\begin{array}{l}0,19 \mathrm{a} \\
0,07 \mathrm{~b}\end{array}$ & $\begin{array}{l}0,19 a \\
0,12 a\end{array}$ & $\begin{array}{l}0,16 a \\
0,12 a\end{array}$ & $\begin{array}{l}0,17 a \\
0,09 a b\end{array}$ & $\begin{array}{l}0,16 a \\
0,11 a\end{array}$ & $\begin{array}{l}-(4) \\
0,12 \mathrm{a}\end{array}$ \\
\hline & Convencional & $\begin{array}{c}0-2^{(1)} \\
10-12^{(3)}\end{array}$ & $\begin{array}{l}0,30 \mathrm{a} \\
0,08 \mathrm{~b}\end{array}$ & $\begin{array}{l}0,30 \mathrm{a} \\
-\end{array}$ & $\begin{array}{l}0,27 a \\
0,13 a b\end{array}$ & $\begin{array}{l}0,26 a \\
0,11 a b\end{array}$ & $\overline{0}, 14 \mathrm{a}$ & $\begin{array}{l}0,20 \mathrm{~b} \\
0,12 \mathrm{ab}\end{array}$ & $\overline{0}, 13 \mathrm{ab}$ \\
\hline \multirow[t]{2}{*}{ Latossolo } & Direto & $\begin{array}{c}0-2^{(1)} \\
10-12^{(1)}\end{array}$ & $\begin{array}{l}0,17 \mathrm{~d} \\
0,10 \mathrm{c}\end{array}$ & $-\overline{0,12 c}$ & $\begin{array}{l}0,36 a b \\
0,44 a\end{array}$ & $\begin{array}{l}0,37 a \\
0,39 a\end{array}$ & $\begin{array}{l}0,31 \mathrm{abc} \\
0,31 \mathrm{~b}\end{array}$ & $\begin{array}{l}0,28 c \\
0,27 b\end{array}$ & $\begin{array}{l}0,29 b c \\
0,25 b\end{array}$ \\
\hline & Convencional & $\begin{array}{c}0-2^{(1)} \\
10-12^{(2)}\end{array}$ & $\begin{array}{c}-(4) \\
-\end{array}$ & $\begin{array}{l}0,19 \mathrm{~b} \\
0,10 \mathrm{~b}\end{array}$ & $0,23 a b$ & $\begin{array}{l}0,34 a \\
0,18 a\end{array}$ & $\begin{array}{l}0,34 a \\
0,26 a\end{array}$ & $\begin{array}{l}0,31 a b \\
0,23 a\end{array}$ & $\begin{array}{l}0,26 a b \\
0,22 a\end{array}$ \\
\hline
\end{tabular}

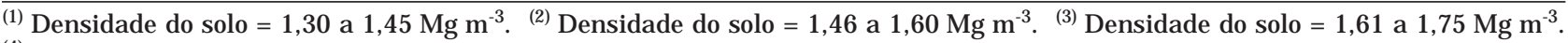
(4) Não determinado. Médias seguidas da mesma letra, na linha, não diferem entre si pelo teste DMS a 5\%. Na construção deste quadro, foram utilizados 260 testes de compressibilidade.

Assim, o aumento do teor de água, expresso pelo grau de saturação, deveu-se aos menores val ores de $\sigma_{p}^{\prime}$, ocasionando deformações plásticas com pequenas pressões aplicadas no solo. No plantio direto, os valores de $D_{s}$ foram normalmente maiores do que no preparo convencional, razão por que a compressão do solo não foi tão intensa quanto no preparo convencional. Entretanto, cuidado deve ser tomado para evitar o tráfego excessivo sobre o solo úmido, especialmente em solos argilosos.

\section{CONCLUSÕES}

1. O índice de compressão mostrou-se reduzido com o aumento da densidade do solo e foi menos influenciado pel o grau de saturação.

2. A maior susceptibilidade do sol o à compressão para oArgissoloVermelho-Amarelo distrófico arênico ocorreu em menor grau de saturação do que em Latossol oVermel ho distrófico típico textura argil osa.

3. O Latossol o Vermel ho distrófico típico textura argilosa apresentou maior capacidade de suportar cargas (maior pressão de preconsolidação) ou foi mais resistenteaté oinício da deformação plástica do queo ArgissoloVermel ho-Amarel o distrófico arênico, porém foi mais susceptível (maior índice de compressão) à compactação ou à deformação plástica.

\section{LITE RATURA CITADA}

ASSOCIAÇÃO BRASILEIRA DE NORMASTÉCNICAS - ABNT. Ensaio de adensamento unidimensional. NBR-12007. Rio de J aneiro, 1990. 13p.
CAMARGO, O.A. \& ALLEONI, L.R.F. Compactação do solo e o desenvolvimento das plantas. Piracicaba, Edição dos Autores, 1997. 133p.

CULLEY, J.L.B. \& LARSON, W.E. Susceptibility to compression of a Clay Loam Haplaquoll. Soil Sci. Soc. Am. J., 51:562567, 1980.

DIAS J UNIOR, M.S. Compression of three soils under longterm tillage and wheel traffic. Michigan, East Lansing State University, 1994. 114p. (Tese de Doutorado)

HÅKANSSON, I. \& VOORHEES, W.B. Chapter on soil compaction. In: LAL, R.; BLUM, W.H.; VALENTIN, C. \& STEWART, B.A., eds. Methods for assessment of soil degradation. Boca Raton, Lewis Publishers, 1997. 576p.

HILLEL, D. Fundamentals of soil physics. Amherst, University of Massachusetts. Academic Press, 1980. 413p.

HOLTZ, R.D. \& KOVACS, W.D. An introduction to geotechnical engineering. New J ersey, Prentice-Hall, 1981. 733p.

KONDO, M.K. \& DIAS J UNIOR, M.S. Compressibilidade de três latossolos em função da umidade e uso. R. Bras. Ci. Solo, 23:211-218, 1999a.

KONDO, M.K. \& DIAS J UNIOR, M.S. Efeito do manejo e da umidade no comportamento compressivo de três latossolos. R. Bras. Ci. Solo, 23:497-506, 1999b.

KONDO, M.K. \& DIAS J UNIOR, M.S. Estimativa do Efeito do uso e da umidade do sol o sobre a compactação adicional de três latossolos. R. Bras. Ci. Solo, 23:773-782, 1999c.

LARSON, W.E.; GUPTA, S.C. \& USECHE, R.A. Compression of agricultural soils from eight soil orders. Soil Sci. Soc. Am. J., 44:450-457, 1980.

PABIN, J .; LIPIEC, J .; WLODEK, S.; BISKUSPSKI, A. \& KAUS, A. Critical soil bulk density and strength for pea seedling root growth as related to other soil factors. Soil Till. Res., 46:203-208, 1998. 
REINERT, D.J. Soil structural form and stability induced by tillage in a typic hapludalf. Michigan, Michigan State University. 1990. 128p. (Tese de Doutorado)

SAS INSTITUTE. SAS U ser's guide: statistics. 5.ed. Cary, N.C., 1985. 956p.

SILVA, R.B.; LIMA, J.M. \& DIAS J UNIOR, M.S. Efeito da adsorção de fosfato em parâmetros físicos e na compressibilidade de solos tropicais. R. Bras. Ci. Solo, 23:219-226, 1999.
SILVA, V.R.; REINERT, D.J . \& REICHERT, J.M. Fatores controladores da compressibilidade dos solos Argissolo Vermel ho-Amarel o distrófico arênico e Latossol o Vermel ho distróficotípico. I - Estado inicial de compactação. R. Bras. Ci. Solo, 26:1-8, 2002.

SOANE, B.D. \& van OUWERKERK, C. Soil compaction problems in world agriculture. In: SOANE, B.D. \& van OUWERKERK, C., eds. Soil compaction in crop production. Amesterdam, Elsevier, 1994. p.1-21. 
V.R. SILVA et al.

R. Bras. Ci. Solo, 26:9-15, 2002 